Acta Crystallographica Section F

Structural Biology

and Crystallization

Communications

ISSN 1744-3091

\section{Zheng Fan, ${ }^{\text {a* Jianxun }} \mathbf{Q i}{ }^{b}$ Yawei Shic and Yiwei Liu ${ }^{\mathrm{a}}$}

${ }^{a}$ Core Facility Center, Institute of Microbiology, Chinese Academy of Sciences, Beijing 100101, People's Republic of China, ${ }^{\mathbf{b}} \mathrm{CAS}$ Key Laboratory of Pathogenic Microbiology and Immunology, Institute of Microbiology, Chinese Academy of Sciences, Beijing 100101, People's Republic of China, and 'Institute of Biotechnology, Key Laboratory of Chemical Biology and Molecular Engineering of the National Ministry of Education, Shanxi University, 92 Wucheng Road, Taiyuan, Shanxi 030006, People's Republic of China

Correspondence e-mail: fanzh@im.ac.cn

Received 18 November 2010

Accepted 22 February 2011

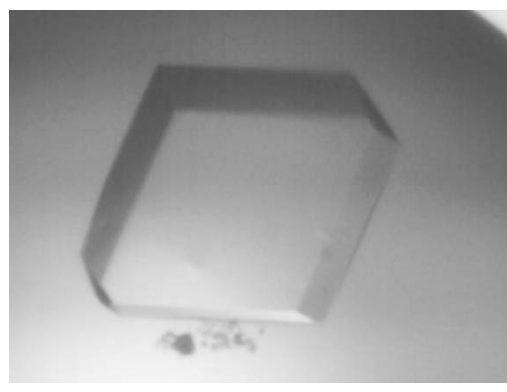

(C) 2011 International Union of Crystallography All rights reserved

\section{Crystallization and preliminary $\mathrm{X}$-ray diffraction analysis of cyclic imide hydrolase $(\mathrm{CIH})$ from Pseudomonas putida YZ-26}

A recombinant form of cyclic imide hydrolase from Pseudomonas putida YZ-26 has been crystallized by the hanging-drop method. X-ray diffraction data were collected to $1.9 \AA$ resolution. The crystals belonged to the orthorhombic space group $C 222_{1}$, with unit-cell parameters $a=111.91, b=176.04, c=176.06 \AA$. Assuming the presence of four molecules in the asymmetric unit gives a $V_{\mathrm{M}}$ value of $3.10 \AA^{3} \mathrm{Da}^{-1}$ and a solvent content of $60.31 \%$.

\section{Introduction}

Cyclic imide hydrolase ( $\mathrm{CIH}$ ), also named imidase, belongs to the cyclic amidohydrolase superfamily. Cyclic amidohydrolases (cyclic amidases; EC 3.5.2.-) have been shown to participate in pyrimidine metabolism in vivo and in the bioconversion of organic acids in vitro, which is critical for producing semisynthetic antibiotics, pesticides and food additives (Soong et al., 2001). According to the characteristics of their optimal substrates, the cyclic amidohydrolase superfamily may be divided into two subfamilies: cyclic ureide hydrolases and cyclic imide hydrolases. The former includes hydantoinase, dihydropyrimidinase, allantoinase and dihydroorotase, while the latter only includes cyclic imide hydrolase, which differs from the former enzymes in the molecular mass of the subunit, amino-acid sequence and substrate specificity.

In 1996, Ogawa and coworkers obtained an imidase from Blasterbacter sp. A17p-4, which was the first time the enzyme had been purified from a bacterium. The imidase participates in the metabolism of simple imides: it is involved in ring-opening hydrolysis of cyclic imides to half-amides and the resulting half-amide is hydrolyzed by halfamidase to a dicarboxylate, which then undergoes further transformation through tricarboxylic acid (TCA) cyclic reactions (Ogawa et al., 1996, 1997). According to these reports, the imidase is a metal-dependent enzyme and is a homogenous trimer with a subunit molecular weight of $35 \mathrm{kDa}$ (Ogawa et al., 1996). Another CIH from pig liver is a homogenous hexamer with a subunit molecular weight of $51 \mathrm{kDa}$ and is also a metal-dependent enzyme (Yang et al., 1993). We obtained a further CIH from Pseudomonas putida YZ-26 (Shi et al., 2007); it is also a zinc-dependent enzyme (Shi et al., 2010).

In order to understand the role of $\mathrm{CIH}$ in pyrimidine metabolism and organic acid bioconversion, we cloned the gene for this $\mathrm{CIH}$ enzyme and expressed the enzyme in Escherichia coli. This is the first report of the crystallization of this $\mathrm{CIH}$ and its preliminary analysis by X-ray crystallography.

\section{Materials and methods}

\subsection{Expression and purification}

The CIH gene was cloned into the plasmid pET32a vector (Shi et al., 2007). The recombinant plasmid $\mathrm{pEI}$ was transformed into E. coli strain BL21 (DE3). The cells were grown in LB medium supplemented with $100 \mu \mathrm{g} \mathrm{ml}^{-1}$ ampicillin at $310 \mathrm{~K}$ until the $\mathrm{OD}_{600}$ 
reached 0.6-0.8. Recombinant protein expression was induced by adding $0.25 \mathrm{~m} M$ isopropyl $\beta$-D-1-thiogalactopyranoside (IPTG) for $5 \mathrm{~h}$. Cells were harvested by centrifugation at $4000 \mathrm{~g}$ for $10 \mathrm{~min}$ and the cell pellets were resuspended in lysis buffer $(500 \mathrm{~m} M \mathrm{NaCl}$, $20 \mathrm{~m} M$ Tris-HCl, $5 \mathrm{~m} M$ imidazole $\mathrm{pH} 8.0$ ) and lysed by sonication. The cell lysate was centrifuged at $12000 \mathrm{~g}$ for $45 \mathrm{~min}$ at $277 \mathrm{~K}$. The supernatant was loaded onto a nickel-affinity column (Qiagen) preequilibrated in lysis buffer and the column containing the His-CIH protein was washed with lysis buffer followed by washing buffer (500 $\mathrm{m} M \mathrm{NaCl}, 20 \mathrm{~m} M$ Tris- $\mathrm{HCl}, 100 \mathrm{~m} M$ imidazole $\mathrm{pH}$ 8.0). The protein was eluted with elution buffer $(20 \mathrm{~m} M$ Tris- $\mathrm{HCl}$ pH 8.0, $500 \mathrm{~m} M \mathrm{NaCl}, 100 \mathrm{~m} M$ EDTA). The eluate was immediately ultrafiltrated to remove the EDTA. After concentration, the preparation was loaded onto a HiLoad 16/60 Superdex 200 pg column (Amersham Biosciences, New Jersey, USA) equilibrated with gel-filtration buffer (50 $\mathrm{m} M$ Tris- $\mathrm{HCl} \mathrm{pH}$ 8.0, $100 \mathrm{mM} \mathrm{NaCl})$. Fractions containing $\mathrm{CIH}$ protein were pooled and concentrated to a concentration of $15 \mathrm{mg} \mathrm{ml}^{-1}$ using Amicon Ultra-15 filters with a $10000 \mathrm{Da}$ cutoff (Millipore, USA).

DLS measurements were carried out at $298 \mathrm{~K}$ using a DynaPro Titan instrument (Wyatt Technology, Santa Barbara, California, USA). Protein concentrations were determined using the BCA protein assay according to the manufacturer's instructions (Pierce).

\subsection{Crystallization}

Crystallization screening was carried out with Crystal Screen and Crystal Screen 2 (Hampton Research). Crystallization trials for CIH were performed at $289 \mathrm{~K}$ using the hanging-drop vapour-diffusion method by mixing $1 \mu \mathrm{l} 5 \mathrm{mg} \mathrm{m}^{-1}$ protein (in buffer consisting of $50 \mathrm{~m} M$ Tris- $\mathrm{HCl} \mathrm{pH} 8.0$ and $100 \mathrm{~m} M \mathrm{NaCl}$ ) and $1 \mu \mathrm{l}$ reservoir solution. The reservoir volume was $200 \mu$ l. Crystals appeared within $7 \mathrm{~d}$ in several different conditions. Diffraction-quality crystals were obtained using the following conditions: $0.1 \mathrm{M}$ bicine $\mathrm{pH} 9.0,2 \%(v / v)$ 1,4-dioxane, $10 \%(w / v)$ polyethylene glycol 20000.

\subsection{Data collection and processing}

The crystals were first transferred into cryoprotectant containing $15 \%$ glycerol for about $30 \mathrm{~s}$ and were then mounted on nylon loops

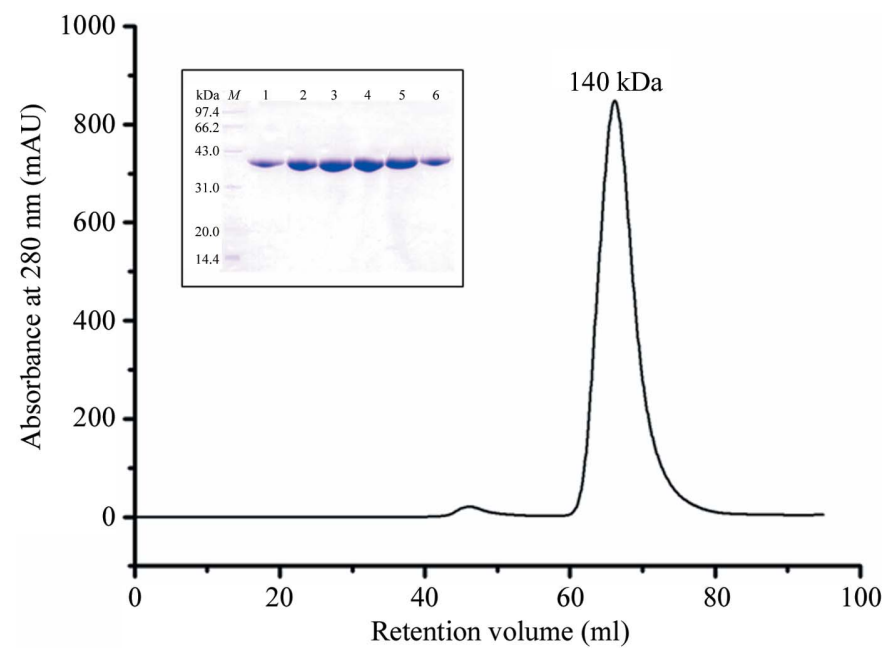

Figure 1

Gel-filtration chromatogram of recombinant $\mathrm{CIH}$ using a HiLoad 16/60 Superdex $200 \mathrm{pg}$ column and SDS-PAGE of the CIH eluted from the gel-filtration column (inset). and flash-cooled in a stream of gaseous nitrogen at $100 \mathrm{~K}$. Diffraction data were collected using an in-house X-ray source (Rigaku MicroMax-007 desktop rotating-anode X-ray generator with a $\mathrm{Cu}$ target operated at $40 \mathrm{kV}$ and $30 \mathrm{~mA}$ ) and an R-AXIS $\mathrm{IV}^{++}$imagingplate detector at a wavelength of $1.5418 \AA$. The collected intensities were indexed, integrated, corrected for absorption, scaled and merged using $H K L-2000$ (Minor et al., 2000).

\section{Results and discussion}

The gene encoding CIH from $P$. putida YZ-26 (GenBank AAY98498.1) was cloned and the protein was overexpressed and purified. The open reading frame for $\mathrm{CIH}$ is $882 \mathrm{bp}$ coding for 293 amino-acid residues. The purified $\mathrm{CIH}$ showed a single band of $35 \mathrm{kDa}$ on SDS-PAGE (Fig. 1), which is in agreement with the calculated molecular weight with a $6 \times$ His tag and a thrombin site (MHHHHHHSSGLVPRGS) at the N-terminus. During gel filtration on a Superdex $200 \mathrm{pg}$ column CIH eluted at the volume expected for a molecular weight of about $140 \mathrm{kDa}$ (Fig. 1). This result is consistent with the DLS experiments (Fig. 2), suggesting that the protein exists as a tetramer in solution.

Crystals appeared within $7 \mathrm{~d}$ (Fig. 3) and an X-ray diffraction image is shown in Fig. 4. The crystals belonged to space group $C 222_{1}$,

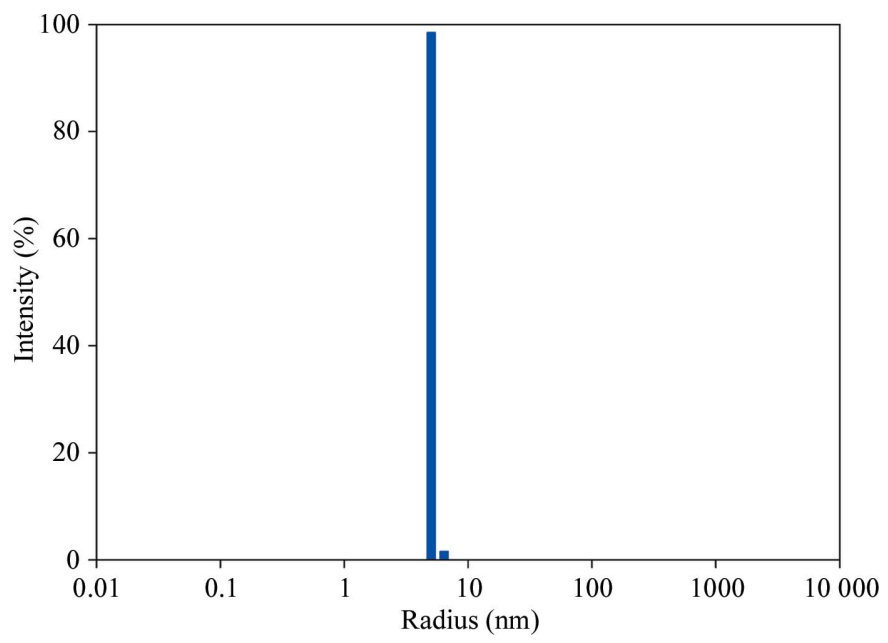

Figure 2

DLS experiment result, showing a single peak of about $140 \mathrm{kDa}$; the molecular radius is $5.0 \mathrm{~nm}$ and the polydispersity is $3.9 \%$.

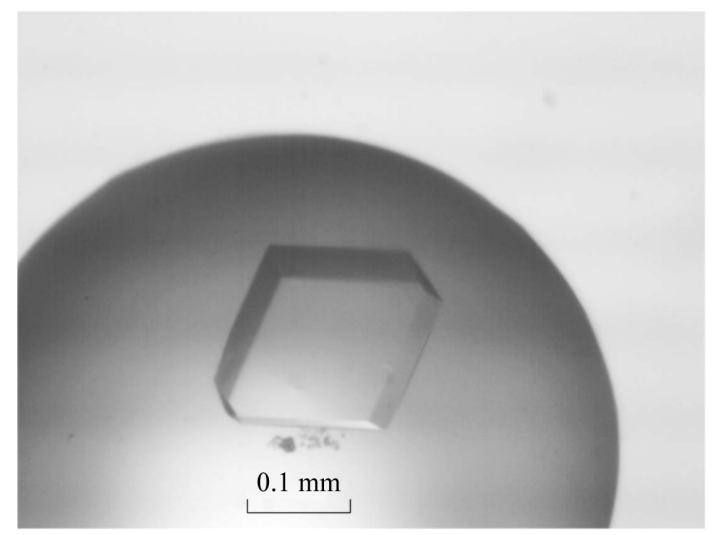

Figure 3

Representative crystal of CIH from P. putida YZ-26. 
Table 1

Data-collection and processing statistics.

Values in parentheses are for the highest resolution shell.

\begin{tabular}{ll}
\hline Space group & $C 222_{1}$ \\
Unit-cell parameters $\left(\AA,^{\circ}\right)$ & $a=111.91, b=176.04, c=176.06$, \\
& $\alpha=\beta=\gamma=90$ \\
Resolution range $(\AA)$ & $50-1.9(1.97-1.90)$ \\
No. of unique reflections & 135717 \\
No. of observed reflections & 861080 \\
Completeness $(\%)$ & $99.7(100.0)$ \\
Multiplicity & $36.3(6.3)$ \\
$\langle I / \sigma(I)\rangle$ & $18.3(9.1)$ \\
$R_{\text {merge }} \dagger(\%)$ & $0.09(0.23)$ \\
\hline
\end{tabular}

$\dagger R_{\text {merge }}=\sum_{h k l} \sum_{i}\left|I_{i}(h k l)-\langle I(h k l)\rangle\right| / \sum_{h k l} \sum_{i} I_{i}(h k l)$.

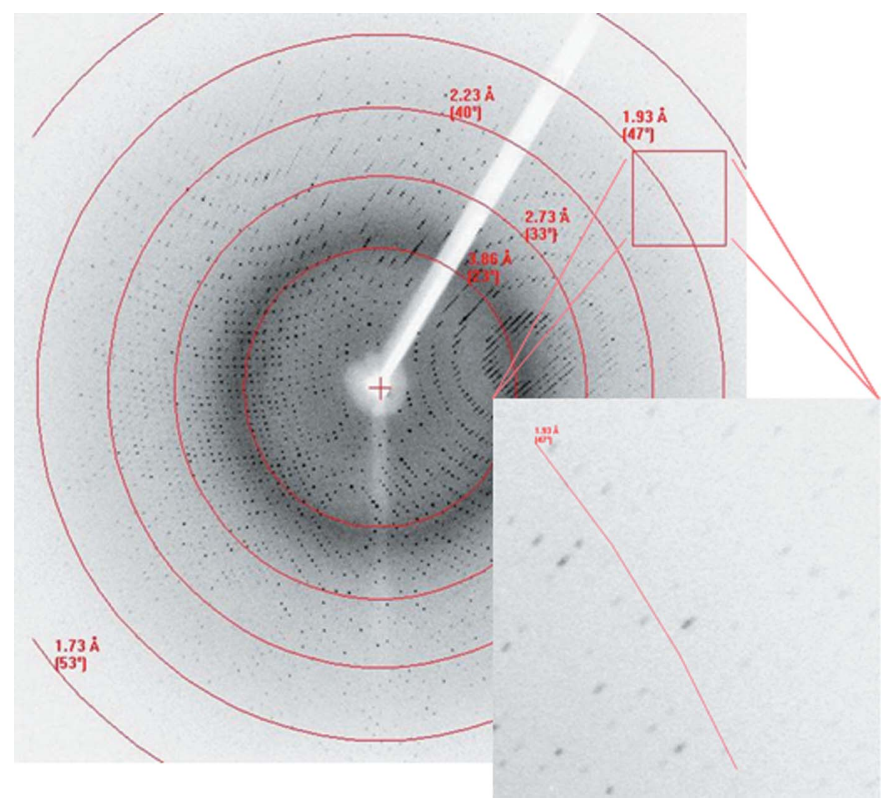

Figure 4

X-ray diffraction image of $\mathrm{CIH}$ from $P$. putida YZ-26. The frame edge is at $1.9 \AA$ resolution. with unit-cell parameters $a=111.91, b=176.04, c=176.06 \AA$, $\alpha=\beta=\gamma=90^{\circ}$. Using the molecular weight of the monomer (35 kDa), a Matthews coefficient (Matthews, 1968) of $3.10 \AA^{3} \mathrm{Da}^{-1}$, corresponding to a solvent content of $60.31 \%$, was obtained assuming the presence of four monomers in the asymmetric unit. Datacollection and processing statistics are given in Table 1. As CIH has $29 \%$ sequence identity to the PuuE allantoinase (PDB entry 3cl6) from P. fluorescens (Ramazzina et al., 2008), we attempted molecularreplacement methods for phase determination. However, this approach did not provide sufficient phases for structure determination. As there are 12 Met residues in the sequence, we are currently preparing selenomethionine-substituted crystals for phase determination using the multiwavelength anomalous diffraction (MAD) technique.

This work was supported by grants from the National Natural Science Foundation of China (grant No. 30871881) and National Basic Research Program 973 (grant No. Y01J021711).

\section{References}

Matthews, B. W. (1968). J. Mol. Biol. 33, 491-497.

Minor, W., Tomchick, D. \& Otwinowski, Z. (2000). Structure, 8, R105-R110.

Ogawa, J., Soong, C.-L., Honda, M. \& Shimizu, S. (1996). Appl. Environ. Microbiol. 62, 3814-3817.

Ogawa, J., Soong, C.-L., Honda, M. \& Shimizu, S. (1997). Eur. J. Biochem. 243, 322-327.

Ramazzina, I., Cendron, L., Folli, C., Berni, R., Monteverdi, D., Zanotti, G. \& Percudani, R. (2008). J. Biol. Chem. 283, 23295-23304.

Shi, Y.-W., Cui, L.-F. \& Yuan, J.-M. (2007). Curr. Microbiol. 55, 61-64.

Shi, Y.-W., Liu, X.-Q., Shi, P. \& Zhang, X.-Y. (2010). Arch. Biochem. Biophys. 494, 1-6.

Soong, C.-L., Ogawa, J. \& Shimizu, S. (2001). Biochem. Biophys. Res. Commun. 286, 222-226.

Yang, Y.-S., Ramaswamy, S. \& Jakoby, W. B. (1993). J. Biol. Chem. 268, 1087010875. 